\title{
Endoscopic full-thickness resection in the colorectum: a single-center case series evaluating indication, efficacy and safety
}

(ㄷ)(1) $(2) \div$

\author{
Authors \\ Institutions \\ 1 Department of Gastroenterology and Hepatology, \\ Northwest Hospital group, Alkmaar, The Netherlands \\ 2 Department of Pathology, Symbiant, Pathology Expert \\ Centre/Northwest Hospital group, Alkmaar, The \\ Netherlands
}

Bas van der Spek ${ }^{*}, 1$, Krijn Haasnoot ${ }^{*}, 1$, Christof Meischl' ${ }^{2}$, Dimitri Heine ${ }^{1}$

submitted 9.2.2018

accepted after revision $\quad 4.7 .2018$

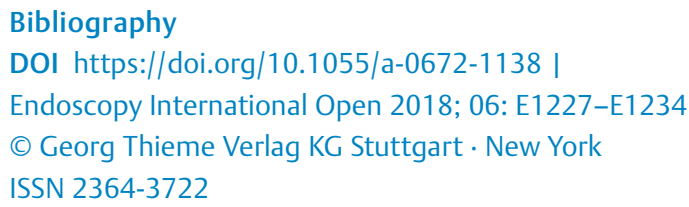

Corresponding author

Dr. B.W. van der Spek, Department of Gastroenterology and Hepatology, Northwest Hospital group, Alkmaar, Wilhelminalaan 12, 1815 JD Alkmaar, The Netherlands Fax: +0031224532199

b.w.vander.spek@nwz.nl

\section{ABSTRACT}

Background and study aims Endoscopic full-thickness resection (eFTR) allows en-bloc and transmural resection of colorectal lesions for which other advanced endoscopic techniques are unsuitable. We present our experience with a novel "clip first, cut later" eFTR-device and evaluate its indications, efficacy and safety.
Patients and methods From July 2015 through October 2017, 51 eFTR-procedures were performed in 48 patients. Technical success and R0-resection rates were prospectively recorded and retrospectively analyzed.

Results Indications for eFTR were non-lifting adenoma ( $\mathrm{n}$ $=19$ ), primary resection of malignant lesion $(n=2)$, resection of scar tissue after incomplete endoscopic resection of low-risk T1 colorectal carcinoma $(n=26)$, adenoma involving a diverticulum $(n=2)$ and neuroendocrine tumor $(n=$ 2). Two lesions were treated by combining endoscopic mucosal resection and eFTR. Technical success was achieved in 45 of 51 procedures ( $88 \%$ ). Histopathology confirmed fullthickness resection in 43 of 50 specimens (86\%) and radical resection (R0) in 40 procedures (80\%). eFTR-specimens, obtained for indeterminate previous T1 colorectal carcinoma resection, were free of residual carcinoma in 25 of 26 cases $(96 \%)$. In six patients ( $13 \%$ ) a total of eight adverse events occurred within 30 days after eFTR. One perforation occurred, which was corrected endoscopically. No emergency surgery was necessary.

Conclusion In this study eFTR appears to be safe and effective for the resection of colorectal lesions. Technical success, R0-resection and major adverse events rate were reasonable and comparable with eFTR data reported elsewhere. Mean specimen diameter $(23 \mathrm{~mm})$ limits its use to relatively small lesions. A clinical algorithm for eFTR case selection is proposed. eFTR ensured local radical excision where other endoscopic techniques did not suffice and reduced the need for surgery in selected cases.

\section{Introduction}

Endoscopic full-thickness resection (eFTR) is a recently introduced minimally invasive endoscopic technique for resecting non-lifting or difficult-to-reach colorectal lesions in which other advanced techniques, such as endoscopic mucosal resection (EMR) or endoscopic submucosal dissection (ESD), are un-

\footnotetext{
* These authors contributed equally.
}

successful [1-3]. eFTR may be an alternative to surgery in selected cases of both benign and malignant colorectal lesions. It has evolved significantly since its introduction and several specific techniques coexist [3]. The single-step eFTR device from Ovesco Endoscopy AG (FTRD) uses a modified over-thescope-clip (OTSC) which is placed below the lesion prior to removing a circular transmural specimen. This technique is also referred to as "clip first, cut later" and allows en-bloc resection, determination of both depth-of-invasion and radicality of re- 
section, while minimizing the risk of fecal spill [1-3]. The procedure is performed in the endoscopy suite, under procedural sedation, with a short hospital stay. The available data concerning eFTR suggest a good safety and efficacy profile [2,4-6]. We aim to present our experiences with FTRD and evaluate eFTR indication, efficacy and safety.

\section{Patients and methods}

\section{Study design}

Data from all eFTR procedures performed for colorectal lesions in adult patients ( $\geq 18$ years) in our hospital from July 2015 through October 2017 were prospectively collected using Castor EDC and retrospectively analyzed [7]. Primary outcome parameter was technical success defined as both en-bloc and macroscopically radical resection of the lesion. Secondary outcome parameters were: $\mathrm{R} 0$ rate defined as free resection margins or no detectable residual lesion in the pathology report, resection specimen size, transmural resection (muscularis propria fully present, including serosal layer), margins free of scar tissue (if applicable), length of hospital stay, adverse events within 30 days as described previously [8] and recurrent lesion and/or clip presence at surveillance endoscopy. Patients were included according to intention-to-treat principles and the acquired data concerning patient characteristics, outcomes, and adverse events (AEs) were analyzed accordingly. The procedure was excluded from the histopathological analysis if no specimen was obtained.

\section{Case selection}

Informed consent was obtained. All patients agreed to undergo eFTR, accepted peri-procedural risks and allowed coded data to be collected for research purposes. Patients undergoing eFTR for a suspected T1 colorectal carcinoma (T1 CRC) or resection of scar tissue from a low-risk T1 CRC were extensively informed about the possibility of existing and remaining lymph node metastases ( $\pm 5 \%$ ) and this was balanced against the risk of undergoing an oncologic resection.

To reach consensus on eFTR indication, all patients with benign or T1 CRC lesions were discussed within our gastroenterology department or multidisciplinary oncology meeting respectively. Tumors were classified as T1 according to the TNM-staging system (tumor invades submucosa, but not muscularis propria). All cases with suspected or confirmed T1 CRC underwent standard dissemination work-up for colorectal cancer, in compliance with current national guidelines. In all patients other available endoscopic resection techniques were deemed insufficient. Cases in which eFTR did not seem feasible (e. g. because of a large lesion or suspected T-stage $>\mathrm{T} 1$ ) were not included in the database. T1 CRC was treated with eFTR either because of suspected low-risk T1 based on endoscopic features as described by Vleugels et al [9] (treatment-naïve) or because of a low-risk T1 based on earlier pathologic findings after initial polypectomy (no lymphovascular invasion, good/moderate differentiation grade, invasion restricted to submucosa) in which only the criterion of a $>1 \mathrm{~mm}$ tumor-free resection margin was not met (T1 scar). Depth of submucosal invasion was not eval- uated in most T1 CRC cases and therefore not analyzed. The category of non-lifting adenomas was subdivided in incomplete resected lesions after use of techniques other than eFTR (primary non-lifting), treatment-naïve lesions not suitable for techniques other than eFTR (primary non-lifting) and recurrent lesions identified upon surveillance endoscopy not suitable for techniques other than eFTR (secondary non-lifting). Small neuroendocrine tumors were resected by eFTR if low-grade and small in accordance with national guidelines.

Eligibility for eFTR in selected patients was based on direct inspection during previous endoscopy or after careful examination of third-party endoscopy reports (including photographs) and discussed by two advanced endoscopists (BS and $\mathrm{GH}$ ).

\section{eFTR technique and procedure}

All procedures were performed by $\mathrm{BS}$ and $\mathrm{GH}$, both skilled in EMR/ESD and trained previously in eFTR on an ex-vivo pig model. In all eFTR procedures the FTR-device from Ovesco Endoscopy AG, Tübingen, Germany was used. This non-reusable device is mounted onto the colonoscope and consists of a transparent cap, on which a modified OTSC is preloaded. Through the endoscope's working channel a grasping forceps can be inserted. By manually turning a handwheel (secured to the endoscope's biopsy valve) the release thread (which runs through the endoscopes working channel) allows clip deployment. Inside the distal end of the cap, just beneath the tip, there is a built-in snare, with its sheath running alongside the scope. The FTR-device is shown in $>$ Fig. 1.

The eFTR procedure itself involves several consecutive steps, which are illustrated in $>$ Fig. 2 . First the lesion is identified and marked properly by applying circumferential markings using coagulation current with the provided marking probe. Then, after reintroduction with a mounted device onto the colonoscope, the lesion is grasped and fully brought into the cap. The modified OTSC is then placed below the lesion, capturing all colonic wall layers in its grasp. In the final step, which follows only seconds after the previous, the specimen is snared above the clip, after which it is secured for histopathological examination. After securing the specimen and dismounting the device, the colonoscope is reintroduced to inspect the resection site for radicality, full closure of the defect and possible bleeding. This single-step "clip first, cut later" full-thickness resection technique prevents extraluminal fluid leakage and the built-in snare avoids the need to reposition the endoscope before snaring, increasing chances of complete resection.

A single shot of an intravenous broad-spectrum antibiotic was administered to all patients. Procedural sedation consisted of either midazolam or propofol in combination with alfentanil. Planned procedure time in right- and left-sided interventions was 90 and 60 minutes, respectively. Patients with left-sided eFTR were prescribed polyethylene glycol once daily for 14 days to prevent clip displacement. 


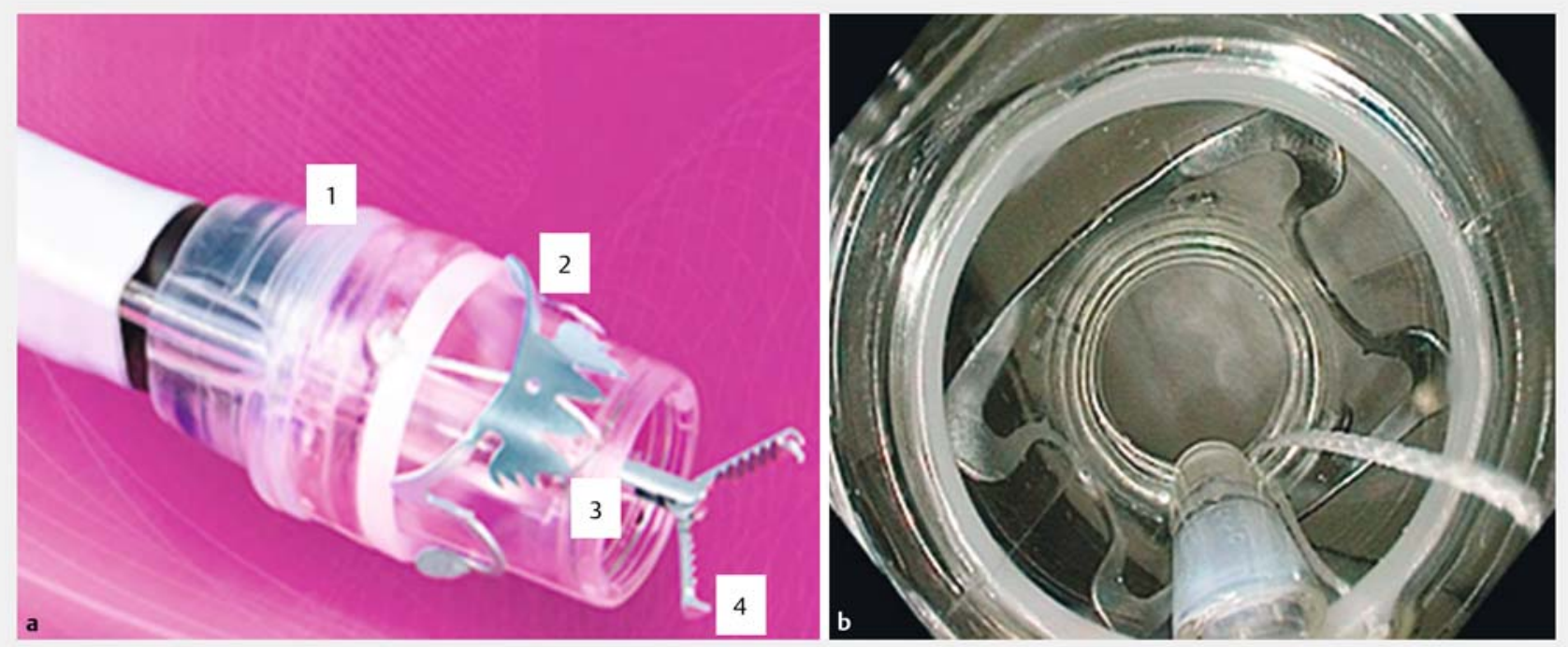

Fig. 1 FTRD (full-thickness resection device) System, Ovesco Endocopy AG. Left: the system consists of a single use transparent cap (1), a modified over-the-scope clip (2), polypectomy-snare (3) and a grasping forceps (4) which is advanced through the colonoscopes working channel. Right: endoscopic view after mounting the FTRD ${ }^{\circledR}$ onto a colonoscope.
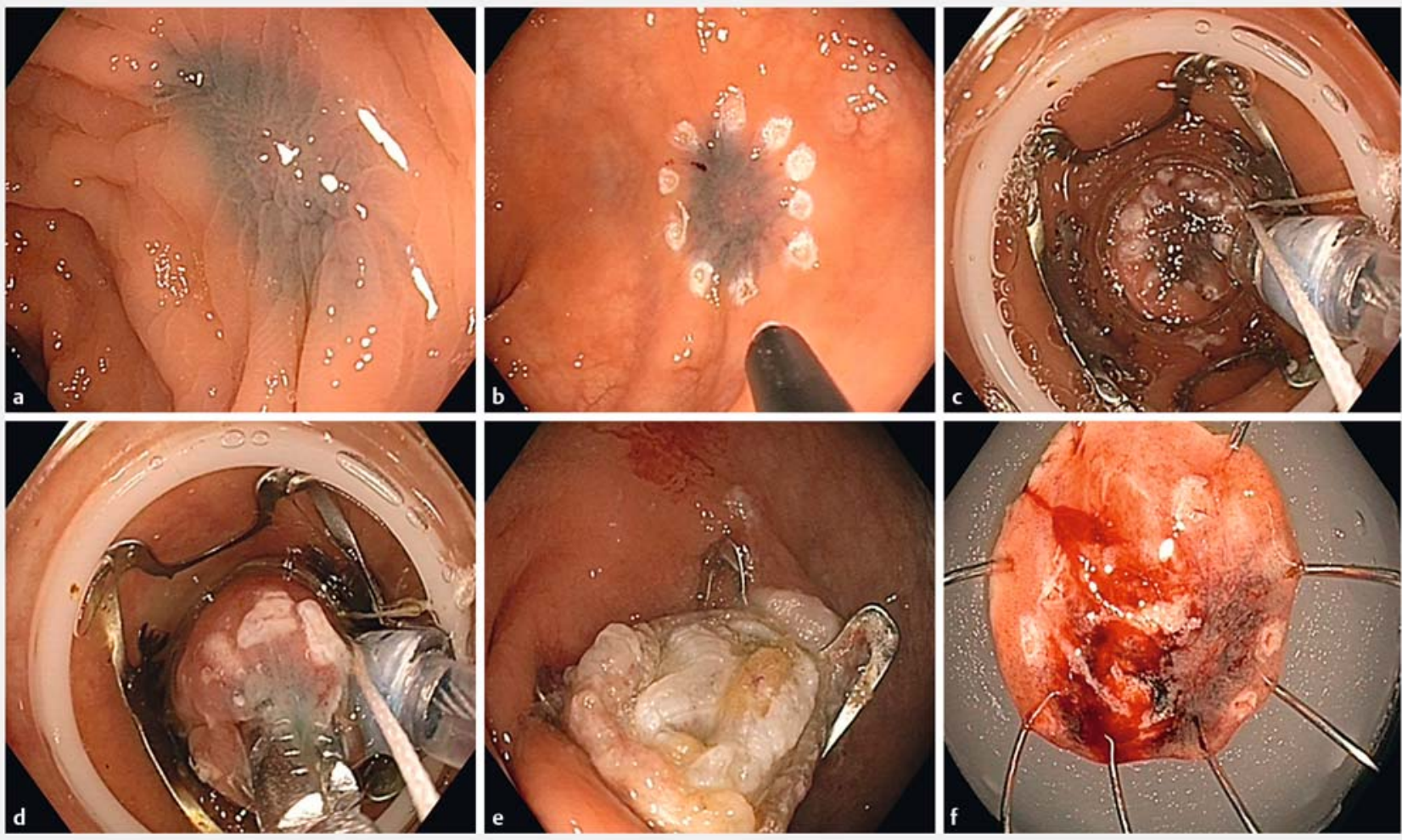

- Fig. 2 eFTR illustration a Scar tissue was identified, previously marked with ink. b The site was marked with marking probe. c After mounting the cap onto the colonoscope (FTRD System) the lesion was identified once more. $\mathbf{d}$ Tissue is pulled into the cap with a grasping forceps. e The modified over-the-scope clip is released and tissue is snared. Inside the clip the muscle layer and peri-colic fat are clearly visible. $\mathbf{f}$ Preparation of the lesion outside the patient. 


\begin{tabular}{|l|c|c|}
\hline Table 1 Patient characteristics. & 48 & $(100)$ \\
\hline Patients, total (\%) & 67 & $(49-76)$ \\
\hline Mean patient age, years (range) & 30 & $(63)$ \\
\hline Male sex, number (\% of total) & 51 & $(100)$ \\
\hline Procedures, total (\%) & & \\
\hline Indication for eFTR, number (\% of total) & 19 & $(37)$ \\
\hline - non-lifting adenoma & 12 & $(24)$ \\
\hline - incomplete endoscopic resection & 4 & $(8)$ \\
\hline - naive & 3 & $(6)$ \\
\hline - recurrent & 28 & $(55)$ \\
\hline - T1-CRC with indication for endoscopic & & \\
\hline resection & 26 & $(51)$ \\
\hline - unclear resection margins & 2 & $(4)$ \\
\hline - suspected submucosal invasion & 2 & $(4)$ \\
\hline - adenoma involving a diverticulum & 12 & $(4)$ \\
\hline - neuroendocrine tumor & 2 & $(16)$ \\
\hline pest invasion into the submucosa according to the international TNM classi- \\
\hline fication; CRC, colorectal cancer
\end{tabular}

\section{Follow-up}

All patients were contacted after eFTR to discuss histopathological results and to inquire about any complications. Endoscopic surveillance was planned 3 months after eFTR to detect recurrence and evaluate clip presence. For further follow-up national guidelines for polyp surveillance were followed, including endoscopic surveillance after 9 months for T1 CRC. Patients referred for additional surgery (step-up approach) were excluded from regular surveillance endoscopy and were treated according to national guidelines concerning follow-up.

\section{Data analysis}

Data were analyzed using SPSS (IBM Corp. Released 2012. IBM SPSS Statistics for Windows, Version 21.0. Armonk, NY: IBM Corp.). Baseline characteristics are displayed descriptively. Categorical variables are displayed both as absolute and relative frequencies (in percentages). For differences in mean spe-
- Table 2 Procedure and specimen characteristics.

\begin{tabular}{|c|c|c|}
\hline $\begin{array}{l}\text { Technical success (macroscopically complete } \\
\& \text { en-bloc), number (\% of total) }\end{array}$ & 45 & $(88)$ \\
\hline - macroscopic incomplete resection ${ }^{1}$ & 5 & $(10)$ \\
\hline - no specimen obtained & 1 & $(2)$ \\
\hline Histology, number (\% of total) & 50 & $(100)$ \\
\hline - confirmed full-thickness resection & 43 & $(86)$ \\
\hline - confirmed complete resection (R0) & 40 & $(80)$ \\
\hline $\begin{array}{l}\text { Histology of T1-CRC after incomplete resec- } \\
\text { tion, number (\% of total) }\end{array}$ & 26 & $(100)$ \\
\hline - lateral and basal margins free of carcinoma & 25 & (96) \\
\hline - lateral and basal margins free of scar tissue & 7 & $(27)$ \\
\hline \multicolumn{3}{|l|}{$\begin{array}{l}\text { Specimen diameter, mean in millimeters } \\
\text { (range) }\end{array}$} \\
\hline - all locations $(n=50)$ & 21 & $(11-45)$ \\
\hline - rectum only $(n=16)$ & 23 & $(11-9)^{2}$ \\
\hline - T1 CRC scar only $(n=26)$ & 23 & $(13-42)^{3}$ \\
\hline \multicolumn{3}{|c|}{$\begin{array}{l}\text { CRC, colorectal cancer; OTSC, over-the-scope clip } \\
1 \text { Case 1: diminutive residual lesion visible within OTSC, no residual lesion at } \\
\text { follow-up endoscopy; case 2: marking visible outside OTSC after resection, } \\
\text { without visible residual lesion, no residual lesion at follow-up endoscopy; } \\
\text { case 3: residual lesion after incomplete eFTR, referral for surgery; case } 4 \text { : } \\
\text { residual lesion after incomplete eFTR, referral for surgery; case 5: diminu- } \\
\text { tive residual lesion visible within OTSC, no residual lesion at follow-up } \\
\text { endoscopy } \\
2 \text { Rectum vs. colon } P=0.147 \\
{ }^{3} \text { T1 CRC scar vs other indications } P=0.889\end{array}$} \\
\hline
\end{tabular}

cimen size between two groups we used an independent samples t-test. $P$ values $<0.05$ were considered significant.

\section{Results}

From July 2015 through October 2017, a total of 51 eFTR procedures were performed in 48 patients (63\% men, median age $69)$. Three patients underwent two separate eFTR procedures for different lesions/locations: two patients with unclear margins of T1 CRC after polypectomy had two scars in close proximity, warranting eFTR of both scars. In a third patient, eFTR was performed on both a non-lifting adenoma and a scar from T1 CRC in one session. eFTR was performed for various indications as described in the Methods section and shown in $>$ Table 1. - Table 2 shows the technical success and R0-resection rates. Five resections were macroscopically incomplete and in one case no specimen could be obtained due to inability to mobilize the lesion into the cap. Mean estimated lesion size was $12.2 \mathrm{~mm}$ (range 2-30). Mean maximum resection specimen diameter was $23 \mathrm{~mm}$ (range $11-45$ ). No significant difference was found in mean specimen diameter in the rectum vs. proximal colon and sigmoid ( 21.0 vs $24.0 \mathrm{~mm} P=0.147$ ) or between scar lesions of T1 CRC vs. other indications (23.1 vs $22.8 \mathrm{~mm}, P=$ $0.889)$. 
Table 3 Adverse events, outcome and surveillance endoscopy.

Adverse events within 30 days, number of 6 patients (\% of total $n=48)^{1}$

\begin{tabular}{|c|c|}
\hline - Minor bleeding & 4 \\
\hline - Major bleeding (transfusion needed) ${ }^{1}$ & 1 \\
\hline - Perforation ${ }^{1}$ & 1 \\
\hline - Postprocedural cardiac event ${ }^{1}$ & 1 \\
\hline - Urinary retention & 1 \\
\hline $\begin{array}{l}\text { Need for surgery, number of patients } \\
(\% \text { of total } n=48 \text { ) }\end{array}$ & 6 \\
\hline
\end{tabular}

- High risk of lymph node metastasis after resection of T1-CRC

\begin{tabular}{|c|c|c|}
\hline - Unsuccessful procedure & 1 & $(2)$ \\
\hline - Recurrent lesion after eFTR & 1 & $(2)$ \\
\hline - Emergency surgery after eFTR & 0 & $(0)$ \\
\hline Mean duration of hospital stay, nights (range) & 1.3 & $(0-8)$ \\
\hline $\begin{array}{l}\text { Surveillance endoscopy, number of patients } \\
(\% \text { of total } n=42)^{2}\end{array}$ & 42 & $(100)$ \\
\hline - Clip in situ & 6 & (14) \\
\hline - Residual or recurrent lesion & 5 & (12) \\
\hline $\begin{array}{l}\text { - Mean time to surveillance endoscopy, days } \\
\text { (SE) }\end{array}$ & 130 & $( \pm 11.9)$ \\
\hline $\begin{array}{l}\text { 2nd surveillance for T1-CRC, number of } \\
\text { patients (\% of total } n=26)^{3}\end{array}$ & 17 & $(65)$ \\
\hline $\begin{array}{l}\text { - No residual or recurrent lesion (\% of total } \\
\mathrm{n}=17 \text { ) }\end{array}$ & 17 & $(100)$ \\
\hline $\begin{array}{l}\text { - Mean time to second surveillance } \\
\text { endoscopy, days (SE) }\end{array}$ & 317 & $( \pm 24.5)$ \\
\hline
\end{tabular}

CRC, colorectal cancer; eFTR, endoscopic full-thickness resection; OSTC, over-the-scope clip

${ }^{1}$ Eight complications occurred in 6 patients. In one patient a per-procedural perforation due to inadequate clip release was closed immediately by OTSC placement with good clinical recovery. On Day 4 an acute coronary artery syndrome was treated with platelet inhibitors, followed by a colonic bleed requiring transfusion.

${ }^{2}$ Lost: after surgical resection $n=5$, patient wish $n=1$.

${ }^{3}$ Lost: after surgical resection $n=4$, comorbidity $n=1$, high-grade dysplasia $n=1$, planned $n=3$.

Histopathology confirmed full-thickness resection in 43 of 50 specimens ( $86 \%$ ) and radical resection (R0) in 40 procedures ( $80 \%)$. Two large lesions $(30 \mathrm{~mm})$ were treated by combining EMR and eFTR in a single session, in both cases radicality could not be confirmed due to the piecemeal resection technique. eFTR specimens obtained from scars of previous indeterminate T1 CRC resection were free of residual carcinoma in 25 of 26 cases (96\%). In one case eFTR of macroscopic unsuspicious scar tissue showed residual adenocarcinoma, with invasion of the muscularis propria (T2). This patient was referred for additional oncologic surgery. Radicality of scar tissue, defined as no fibrous tissue in the lateral and basal resection margins, was confirmed only in 7 of 26 cases (27\%).
In six patients a total of eight AEs occurred ( $\triangleright$ Table 3 ). Four of these patients had minor bleeding not necessitating blood transfusion. No emergency surgery was necessary. Six patients needed additional surgery because of either high risk for lymph node metastases ( $n=4$; three patients with T2-T3 CRC and one patient with lymphatic invasion in T1 CRC), technical failure of eFTR $(n=1)$ or endoscopically untreatable adenoma recurrence at surveillance $(n=1)$. None of the oncologic surgical resections showed residual tumor or lymph node metastases, nor did the surgeon report technical difficulties related to OTSC presence.

Forty-two of 48 patients underwent surveillance endoscopy after a mean period of 130 days ( \pm 11.9 ). In five patients no inspection of eFTR-scar was performed because of surgical resection and one patient wanted no surveillance ( $>$ Table 3 ). Five patients had a residual/recurrent lesion, either treated conservatively $(n=2)$, with additional EMR $(n=2)$ or surgery $(n=1)$.

Second surveillance is available in 17 of 26 patients following eFTR for T1 CRC after a mean period of $317( \pm 24.5)$ days. No local recurrence was detected.

\section{Discussion}

Results from this retrospective case-series describing 51 procedures suggest that eFTR is a feasible and safe option for resecting colorectal lesions, where other advanced endoscopic techniques do not suffice. Technical success (88\%) and R0-resection (80\%) were reasonable and comparable with eFTR-data reported elsewhere [2,4-6].

Minor AEs occurred in five patients (10\%) and major AEs in one patient ( $2 \%)$. We experienced one immediate perforation, caused by a non-releasing OTSC, with the clip-releasing system obscured from view by the lesion itself, illustrating the importance of a clear view on OTSC deployment at all times during the procedure. Fortunately, in this case, the perforation could be corrected endoscopically by OTSC. However, following eFTR, this patient developed acute coronary artery syndrome and subsequently a colonic bleed after introduction of platelet inhibitors. Endoscopic inspection showed no bleeding site and adequate closure of the perforation. Schmidt et al published five cases of immediate and one case of late perforation (3.3\%) confirming the relevance of this complication in eFTR [2]. Complications reported elsewhere, including entrapment of extracolonic organs within the OTSC, such as the small bowel, and acute appendicitis after eFTR of the appendix base did not occur in our series. It is important to state that, although reported in 34 cases by Schmidt and colleagues, in our series no eFTR of lesions at the appendix base was performed [2]. Overall, most complications concerned patient who reported minor bleeding ( $8 \%$ ) that did not necessitate blood transfusion. This is comparable to wide-field EMR and seems acceptable, especially when compared to mortality and morbidity numbers in colorectal surgery, which is considered the alternative procedure in these cases.

Although colonic lumen diameter may appear to be diminished directly after eFTR, no clinically relevant stenosis occurred, nor has this been described elsewhere. The OTSC tended to spontaneously dislocate unnoticed (OTSC in situ in only $14 \%$ 


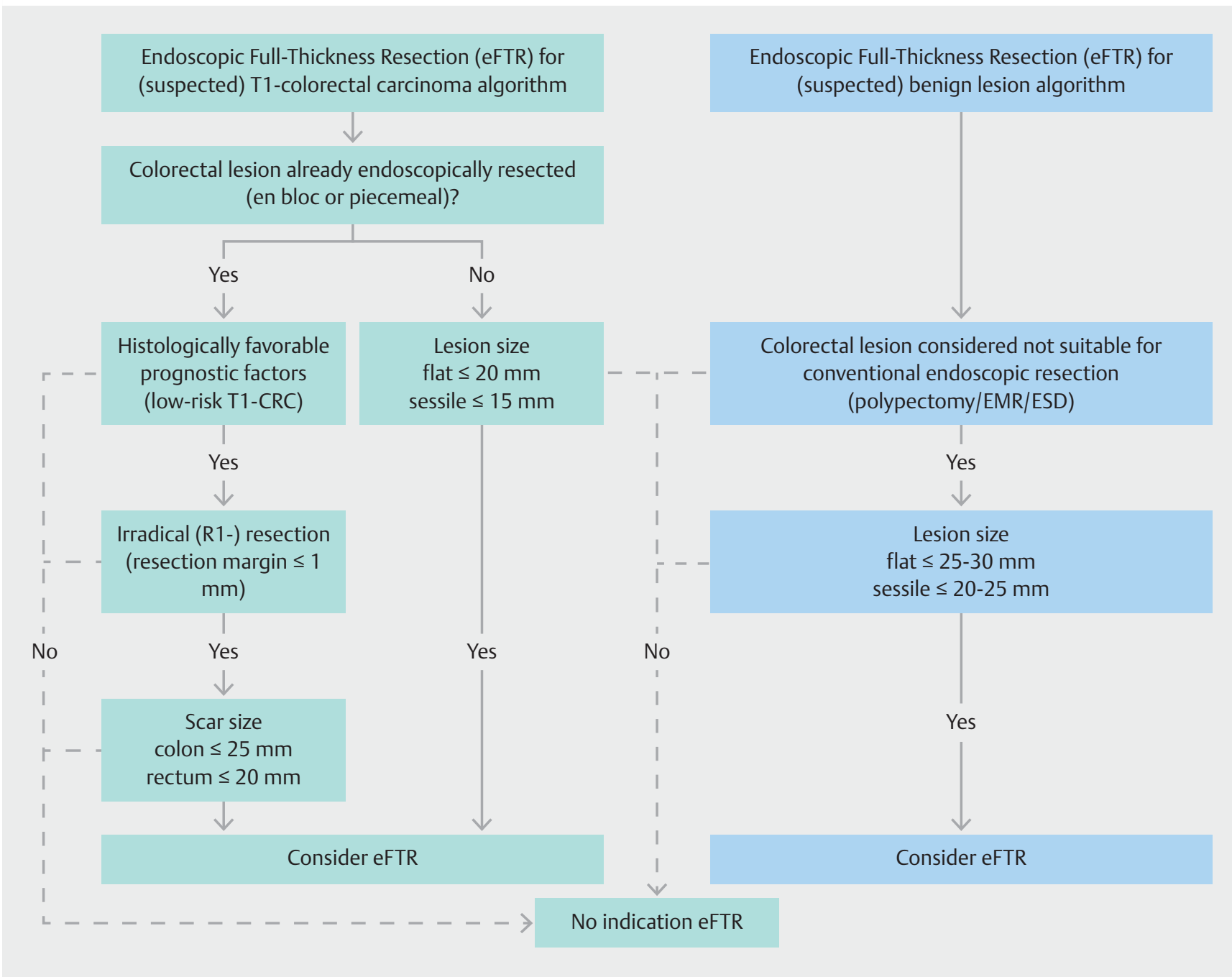

Fig. 3 Proposed clinical algorithm for eFTR in malignant (left) and benign (right) colorectal lesions. It incorporates lesion size, morphology and location. It involves a stepwise approach and may assist the clinician in decision-making on eFTR indication and applicability. In T1 CRC a multidisciplinary consideration, weighing the risk of residual lymph node metastases after eFTR against morbidity and mortality of colorectal surgery, and the informed consent of the patient are mandatory.

Left frame: (suspected) T1-colorectal carcinoma = suspected low-risk T1 CRC based on endoscopic features as described by Vleugels et al [9] or confirmed (low-risk) T1 CRC based on histopathologic findings after initial polypectomy or biopsy; histologically favorable factors = no lymphovascular invasion, good/moderate differentiation grade and invasion restricted to submucosa; irradical resection = the criterium of $>1 \mathrm{~mm}$ tumor-free resection margin was not met; scar size = scar size as estimated by the endoscopist, being no smaller than the initial lesion's base diameter; flat, sessile lesion = lesion morphology according to the Paris endoscopic classification of superficial neoplastic lesions, types 0 -Ila, 0 -IIb, 0-Ilc (flat) and 0-Is (sessile) respectively.

Right frame: (suspected) benign lesion = suspected benign colorectal lesion based on endoscopic features as described by Vleugels et al [9] or confirmed benign lesion based on histopathologic findings after initial incomplete polypectomy or biopsy;

after a mean follow-up of 130 days). Although rarely indicated, a clip cutter device exists allowing endoscopic clip removal [10].

We performed additional eFTR of scar tissue after endoscopic resection of low-risk T1 CRC in cases in which radical resection could not be confirmed microscopically (i.e. after piecemeal resection or $\leq 1 \mathrm{~mm}$ tumor-free resection margin). In 25 of 26 cases, local radical resection was plausible after eFTR (lateral and basal margins free of carcinoma) and surgery could be avoided. In T1 CRC, several histologic features are associated with either low or high risk for lymph node metastases and local recurrence is rare after R0-endoscopic resection [11]. In the Dutch guideline, radical resection, good/moderate differentiation and absence of lymphovascular invasion classify T1 CRC as low-risk. Endoscopic R0-resection is regarded as an adequate treatment strategy for low-risk T1 CRC as oncologic surgical resection has a high number-needed-to-treat and significant morbidity/mortality $[12,13]$.

Overall 30-day mortality and postoperative complicated course rates for oncologic surgery in the Netherlands in 2015 were $2.3 \% / 15 \%$ (colon) and $1.2 \% / 20 \%$ (rectum) respectively [14]. After endoscopic R0-resection of low-risk T1-CRC by con- 
ventional polypectomy the patient and his or her consulting physician have to decide upon the need for additional oncologic surgery. This implies weighing the risk of residual lymph node metastases against morbidity and mortality of colorectal surgery. However, R1 resection after EMR/ESD/polypectomy for otherwise low-risk T1 CRC occurs frequently, warranting additional treatment, often resulting in oncologic surgery. Therefore, safe and reliable minimally invasive endoscopic treatment approaches are needed to lower the burden of treatment for T1 CRC. This is of increasing importance as colorectal cancer screening programs cause a shift towards earlier tumor stages at diagnosis $[15,16]$. eFTR has been reported by others as an option for confirming radical local excision in T1 CRC $[2,4-6$, 17]. In our center, colorectal surgery is performed only for low-risk T1 CRC when eFTR fails to achieve radical local excision. We refer to this two-stage strategy as the "eFTR step-up approach." Although most guidelines require a resection margin $>1 \mathrm{~mm}$ to confirm radicality of resection and to allow endoscopic surveillance for low-risk T1 CRC, no residual tumor was found in $25 / 26$ cases ( $96 \%$ ) with inconclusive radicality in our series. This casts doubts on the relevance of the $>1 \mathrm{~mm}$ margin requirement and the necessity for eFTR, introducing additional complication risks, in all of these cases. Furthermore, histopathological confirmation of complete scar tissue resection was confirmed in only $27 \%$, raising questions about the ability of eFTR to completely resect scar tissue and truly confirm radicality of resection. Therefore, scar size as estimated by the endoscopist, being no smaller than the initial lesion's base diameter, may limit FTRD use in these cases. Nevertheless, we found no recurrence of CRC so far in these patients during follow-up endoscopies.

Adequate case selection is challenging and requires experience and careful pre-procedural evaluation. Mean specimen diameter $(23 \mathrm{~mm})$ limits FTRD use to relatively small lesions. Therefore, we propose a clinical algorithm for eFTR case selection (> Fig. 3).

The eFTR learning curve is unknown. We have experienced that mastering the eFTR-technique has a significantly shorter learning curve than EMR or ESD, but requires proper training, for example on an ex vivo pig model.

This study is limited by its retrospective, single-center nature, introducing possible selection bias and questioning reproducibility of the results. Prospective studies need to clarify the role of eFTR in the treatment of low-risk T1 colorectal cancer, the necessity of antibiotic prophylaxis, the need for post-eFTR hospitalization and need to produce universally accepted treatment algorithms.

\section{Conclusion}

Based on these data, eFTR using this single-step FTR-device seems successful and safe in the colon and rectum. Technical success, R0-resection and major AEs rate were reasonable and comparable with eFTR data reported elsewhere. Mean specimen diameter $(23 \mathrm{~mm})$ limits its use to relatively small lesions. With eFTR gastroenterologists may avoid the need for surgery in selected cases of low-risk T1 colorectal carcinoma, non-lift- ing adenoma, submucosal tumors and lesions in difficult-toreach locations. Prospective studies are necessary to clarify the role of eFTR in the treatment of low-risk T1 colorectal carcinoma, comparing oncological outcome, weighing risk of residual lymph node metastases after eFTR against morbidity and mortality of colorectal surgery. Furthermore, the necessity for antibiotic prophylaxis in a procedure without fecal spill and the need for post-eFTR hospitalization have yet to be determined.

\section{Competing interests}

None

References

[1] Schmidt A, Bauerfeind P, Gubler C et al. Endoscopic full-thickness resection in the colorectum with a novel over-the-scope device: first experience. Endoscopy 2015; 47: 719-725

[2] Schmidt A, Beyna T, Schumacher B et al. Colonoscopic full-thickness resection using an over-the-scope device: a prospective multicentre study in various indications. Gut 2018; 67: $1280-1289$

[3] Schmidt A, Meier B, Caca K. Endoscopic full-thickness resection: Current status. World J Gastroenterol 2015; 21: 9273 - 9285

[4] Richter-Schrag H], Walker C, Thimme R et al. Full thickness resection device (FTRD). Experience and outcome for benign neoplasms of the rectum and colon. Chirurg 2016; 87: 316-325

[5] Andrisani G, Pizzicannella M, Martino M et al. Endoscopic full-thickness resection of superficial colorectal neoplasms using a new overthe-scope clip system: A single-centre study. Dig Liver Dis 2017; 49: $1009-1013$

[6] Valli PV, Mertens ], Bauerfeind P. Safe and successful resection of difficult Gl lesions using a novel single-step full-thickness resection device $\left(\right.$ FTRD $\left.^{\circledR}\right)$. Surg Endosc 2018; 32: 289-299

[7] Castor Electronic Data Capture. Ciwit BV Amsterdam, The Netherlands: 2018

[8] Cotton PB, Eisen GM, Aabakken L et al. A lexicon for endoscopic adverse events: report of an ASGE workshop. Gastrointest Endosc 2010; 71: $446-454$

[9] Vleugels JLA, Hazewinkel Y, Dekker E. Morphological classifications of gastrointestinal lesions. Best Pract Res Clin Gastroenterol 2017; 31: 359-367

[10] Caputo A, Schmidt A, Caca K et al. Efficacy and safety of the remOVE System for OTSC ${ }^{\circledR}$ and FTRD ${ }^{\circledR}$ clip removal: Data from a PMCF analysis. Minim Invasive Ther Allied Technol 2018; 27: 138-142

[11] Bosch SL, Teerenstra S, de Wilt JH et al. Predicting lymph node metastasis in PT1 colorectal cancer: A systematic review of risk factors providing rationale for therapy decisions. Endoscopy 2013; 45: 827 834

[12] Labianca R, Nordlinger B, Beretta GD et al. Early colon cancer: ESMO clinical practice guidelines for diagnosis, treatment and follow-up. Ann Oncol 2013; 24: (Suppl. 06): vi64-72

[13] Backes Y, de Vos Tot Nederveen Cappel WH, van Bergeijk J et al. Risk for incomplete resection after macroscopic radical endoscopic resection of T1 colorectal cancer: A multicenter cohort study. Am J Gastroenterol 2017; 112: 785-796

[14] Van Leersum NJ, Snijders HS, Henneman D et al. The Dutch surgical colorectal audit. Eur J Surg Oncol 2013; 39: 1063-1070

[15] Morris EJ, Whitehouse LE, Farrell T et al. A retrospective observational study examining the characteristics and outcomes of tumours diag- 
nosed within and without of the English NHS Bowel Cancer Screening Programme. Br J Cancer 2012; 107: 757-64

[16] De Neree Tot Babberich MP, van der Willik EM, van Groningen JT et al. Surgery for colorectal cancer since the introduction of the Netherlands national screening programme. Investigations into changes in number of resections and waiting times for surgery. Ned Tijdschr Geneeskd 2017; 161: D997

[17] Lagoussis P, Soriani P, Tontini GE et al. Over-the-scope clip-assisted endoscopic full-thickness resection after incomplete resection of rectal adenocarcinoma. Endoscopy 2016; 48: (Suppl. 01): E59-60

\section{CORRECTION}

van der Spek B, Haasnoot K, Meischl, C et al. Endoscopic full-thickness resection in the colorectum: a single-center case series evaluating indication, efficacy and safety

Endoscopy International Open 2018; 06: E1227-E1234. DOI: 10.1055/a-0672-1138

In the above mentioned article an author name was corrected. Correct is: Dimitri Heine. 